\title{
WEAKLY INFINITE-DIMENSIONAL PRODUCT SPACES
}

\author{
DALE M. ROHM
}

(Communicated by James E. West)

\begin{abstract}
It is shown that the product of a weakly infinite-dimensional compactum with a $C$-space is weakly infinite-dimensional. Some observations on the coincidence of weak infinite-dimensionality and property $C$ are made. The question of when a weakly infinite-dimensional space has weakly infinitedimensional product with all zero-dimensional spaces is investigated.
\end{abstract}

\section{INTRODUCTION}

By a space we mean a metric space, and by the dimension of a space we mean the Lebesgue covering dimension, for example as presented in [E]. In this sequel paper, we continue the line of investigation, initiated in [R2], into when the product of two weakly infinite-dimensional spaces is itself weakly infinite-dimensional. The reader is referred to that source for a more complete discussion of the history of this question and specifically for the definitions of countable-dimensional, weakly infinite-dimensional, and strongly infinite-dimensional spaces, as well as these of property $C, C$-space, and $C$-refinement.

While it is known that every countable-dimensional space, hence every finitedimensional space, has property $C$ and that every $C$-space is weakly infinitedimensional, R. Pol has constructed a compact $C$-space which is not countabledimensional [P1]. It remains unknown whether or not every weakly infinitedimensional space must have property $C$.

Similarly, while it is also known that the product of two $C$-spaces can be strongly infinite-dimensional [EP], [P2], it is still unknown whether or not the product of two weakly infinite-dimensional compacta must always be weakly nfinite-dimensional. On the other hand, the productivity of property $C$ for ompacta has been established [R2].

Theorem. The product of two $C$-spaces, one of which is compact, is itself a $C$ space.

In this paper we investigate the productivity of weak infinite-dimensionality for compacta, showing productivity for a large, perhaps the entire, class of

Received by the editors August 5, 1988 .

1980 Mathematics Subject Classification (1985 Revision). Primary 54F45, 54B10; Secondary $57 \mathrm{~N} 20$.

Key words and phrases. Property $C$, weakly infinite-dimensional spaces, product spaces. 
weakly infinite-dimensional factors. Finally, some observations concerning the coincidence of weak infinite-dimensionality and property $C$ are given, along with some remarks on when a weakly infinite-dimensional space has weakly infinite-dimensional product with all zero-dimensional spaces.

\section{RESULTS AND PROOFS}

In proving our main result, we will make use of the following characterization of weak infinite-dimensionality in terms of binary open covers:

Lemma 1. A space $X$ is weakly infinite-dimensional if and only if, for any sequence of binary open covers $\left\{\mathscr{U}_{n}: n \in \mathbb{N}\right\}$ of $X$ of the form $\mathscr{U}_{n}=\left\{U_{n}^{1}, U_{n}^{2}\right\}$, there exists a precise pairwise disjoint open refinement $\mathscr{V}_{n}$ of each $U_{n}$-i.e.:

1. For each $n \in \mathbb{N}, \mathscr{V}_{n}=\left\{V_{n}^{1}, V_{n}^{2}\right\}$, with $V_{n}^{1}$ and $V_{n}^{2}$ open in $X$.

2. For each $n \in \mathbb{N}, V_{n}^{1} \cap V_{n}^{2}=\varnothing$.

3. For each $n \in \mathbb{N}, V_{n}^{1} \subset U_{n}^{1}$ and $V_{n}^{2} \subset U_{n}^{2}$ - so that the $\bigcup\left\{\mathscr{V}_{n}^{n}: n \in \mathbb{N}\right\}$ forms an open cover of $X$.

As the proof is elementary, involving only complete normality, we omit it. For this characterization and further generalizations of the notion, we refer the reader to [R1, Chapter 3].

Theorem 1. The product of a weakly infinite-dimensional compactum with a $C$ space is again weakly infinite-dimensional.

Proof. Given a $C$-space $X$ and a weakly infinite-dimensional compactum $Y$, we show that the product $X \times Y$ is weakly infinite-dimensional. Let a countable collection of binary open covers of $X \times Y$ be given. We rewrite this collection as a sequence of such countable collections

$$
\left\{\left\{\mathscr{U}_{m, n}: n \in \mathbb{N}\right\}: m \in \mathbb{N}\right\},
$$

where each binary open cover has the form

$$
\mathscr{U}_{m, n}=\left\{U_{m, n}^{1}, U_{m, n}^{2}\right\} .
$$

Fix $m \in \mathbb{N}$, let $x \in X$ be fixed but arbitrary, and let $\pi: X \times Y \rightarrow X$ denote the projection mapping. For each $n \in \mathbb{N}$ and $\alpha \in\{1,2\}$, we set

$$
U_{m, n}^{\alpha}(x)=U_{m, n}^{\alpha} \cap \pi^{-1}(x) \text { and } \mathscr{U}_{m, n}(x)=\left\{U_{m, n}^{1}(x), U_{m, n}^{2}(x)\right\} .
$$

Thus, $\mathscr{U}_{m, n}(x)$ is a binary open cover of $\pi^{-1}(x)$ for each $n \in \mathbb{N}$. Since $\pi^{-1}(x)$ is homeomorphic to $Y$, we will not, when the context is clear, distinguish between $\pi^{-1}(x)$ as a subspace of $X \times Y$, and $Y$.

In particular, $\pi^{-1}(x)$ is weakly infinite-dimensional, so that, using Lemma 1 , we can choose subsets $V_{m, n}^{1}(x)$ and $V_{m, n}^{2}(x)$ of $\pi^{-1}(x)$ for each $n \in \mathbb{N}$ with

1. $V_{m, n}^{1}(x)$ and $V_{m, n}^{2}(x)$ open in $\pi^{-1}(x)$ 
2. $V_{m, n}^{1}(x) \cap V_{m, n}^{2}(x)=\varnothing$

3. $V_{m, n}^{1}(x) \subset U_{m, n}^{1}(x)$ and $V_{m, n}^{2}(x) \subset U_{m, n}^{2}(x)$,

so that $\left\{V_{m, n}^{\alpha}(x): \alpha=1,2, n \in \mathbb{N}\right\}$ is a cover of $\pi^{-1}(x)$. We then use the compactness of $\pi^{-1}(x)$ to extract a finite subcover

$$
\left\{V_{m, n}^{\alpha}(x): \alpha=1,2, n=1, \ldots, r_{m}(x)\right\}
$$

for some positive integer $r_{m}(x)$, and, using normality, we "shrink" the elements of the finite subcover so that

$$
V_{m, n}^{\alpha}(x) \subset \overline{V_{m, n}^{\alpha}(x)} \subset U_{m, n}^{\alpha}(x)
$$

for each $\alpha \in\{1,2\}$ and $n \in\left\{1, \ldots, r_{m}(x)\right\}$.

Next, we use an idea of Dieudonné [D] to construct an open cover of $X$.

Claim. For each $n \in\left\{1, \ldots, r_{m}(x)\right\}$, there is an open neighborhood $W_{m, n}(x)$ of $x$ in $X$ so that, for any $x^{\prime} \in W_{m, n}(x)$ and $\alpha \in\{1,2\}$, the inclusions

$$
V_{m, n}^{\alpha}(x) \subset \overline{V_{m, n}^{\alpha}(x)} \subset U_{m, n}^{\alpha}\left(x^{\prime}\right)
$$

hold.

Indeed if not, then we could choose a sequence $\left(x_{k}, y_{k}\right)$ in $X \times Y$ with $x_{k} \rightarrow x$ where, without loss of generality, for each $k \in \mathbb{N}$,

$$
y_{k} \in \overline{V_{m, n}^{1}(x)} \text { but } y_{k} \notin U_{m, n}^{1}\left(x_{k}\right) \text {. }
$$

By the compactness of $Y$, passing to a convergent subsequence if necessary, we have

$$
y_{k} \rightarrow y \in \overline{V_{m, n}^{1}(x)} \subset U_{m, n}^{1}(x)
$$

so that

$$
\left(x_{k}, y_{k}\right) \rightarrow(x, y) \in U_{m, n}^{1} .
$$

But then, since $U_{m, n}^{1}$ is open in $X \times Y$, we see that, for all sufficiently large $k$,

$$
\left(x_{k}, y_{k}\right) \in U_{m, n}^{1}, \quad \text { so that } y_{k} \in U_{m, n}^{1}\left(x_{k}\right),
$$

which is a contradiction.

We construct such an open set $W_{m, n}(x)$ for each $n \in\left\{1, \ldots, r_{m}(x)\right\}$ and set

$$
W_{m}(x)=\bigcap\left\{W_{m, n}(x): n=1, \ldots, r_{m}(x)\right\} .
$$

Then, $W_{m}(x)$ is an open neighborhood of $x \in X$, so that

$$
\left\{W_{m}(x) \times V_{m, n}^{\alpha}(x): \alpha=1,2, n=1, \ldots, r_{m}(x)\right\}
$$

is an open cover of $\pi^{-1}(x)$ in $X \times Y$. We form the open cover

$$
\mathscr{W}_{m}=\left\{W_{m}(x): x \in X\right\}
$$

of $X$ by constructing such a neighborhood $W_{m}(x)$ for each $x \in X$. 
In this manner, we construct such an open cover $\mathscr{W}_{m}$ of $X$ for each $m \in \mathbb{N}$. Since $X$ has property $C$, we can choose a $C$-refinement $\mathscr{O}_{m}$ of $\mathscr{W}_{m}$ for each $m \in \mathbb{N}$ so that the $\cup\left\{\mathscr{O}_{m}: m \in \mathbb{N}\right\}$ covers $X$. Since each $\mathscr{O}_{m}$ refines $\mathscr{W}_{m}$, we can choose a function $\phi_{m}: \mathscr{O}_{m} \rightarrow X$ for each $m \in \mathbb{N}$ so that if $O \in \mathscr{O}_{m}$ we have

$$
O \subset W_{m}\left(\phi_{m}(O)\right) \text {. }
$$

Thus, if $n \in\left\{1, \ldots, r_{m}\left(\phi_{m}(O)\right)\right\}$ for some $O \in \mathscr{O}_{m}$, then

$$
O \subset W_{m}\left(\phi_{m}(O)\right) \subset W_{m, n}\left(\phi_{m}(O)\right),
$$

so that for $\alpha \in\{1,2\}$ we have

$$
O \times V_{m, n}^{\alpha}\left(\phi_{m}(O)\right) \subset W_{m, n}\left(\phi_{m}(O)\right) \times V_{m, n}^{\alpha}\left(\phi_{m}(O)\right) \subset U_{m, n}^{\alpha} .
$$

For each $m, n \in \mathbb{N}$ and $\alpha \in\{1,2\}$, we define

$$
C_{m, n}^{\alpha}=\bigcup\left\{O \times V_{m, n}^{\alpha}\left(\phi_{m}(O)\right): n \in\left\{1, \ldots, r_{m}\left(\phi_{m}(O)\right)\right\} \text { for some } O \in \mathscr{O}_{m}\right\}
$$

and set

$$
\mathscr{C}_{m, n}=\left\{C_{m, n}^{1}, C_{m, n}^{2}\right\} .
$$

If $(x, y) \in C_{m, n}^{\alpha}$, then there exists $O \in \mathscr{O}_{m}$ with $n \in\left\{1, \ldots, r_{m}\left(\phi_{m}(O)\right)\right\}$ so that

$$
(x, y) \in O \times V_{m, n}^{\alpha}\left(\phi_{m}(O)\right) \subset U_{m, n}^{\alpha} .
$$

Therefore, $\mathscr{C}_{m, n}$ is a precise open refinement of $\mathscr{U}_{m, n}$. Furthermore, since the elements of $\mathscr{\mathscr { O }}_{m}$ are pairwise disjoint, and since

$$
V_{m, n}^{1}\left(\phi_{m}(O)\right) \cap V_{m, n}^{2}\left(\phi_{m}(O)\right)=\varnothing
$$

for any $O \in \mathscr{O}_{m}$ with $n \in\left\{1, \ldots, r_{m}\left(\phi_{m}(O)\right)\right\}$, we see that

$$
C_{m, n}^{1} \cap C_{m, n}^{2}=\varnothing \text {. }
$$

Finally, since $\bigcup\left\{\mathscr{O}_{m}: m \in \mathbb{N}\right\}$ covers $X$, given a point $(x, y) \in X \times Y$ we can find $m \in \mathbb{N}$ and $O \in \mathscr{O}_{m}$ so that $x \in O$. Since $\pi^{-1}\left(\phi_{m}(O)\right)$ is covered by

$$
\left\{V_{m, n}^{\alpha}\left(\phi_{m}(O)\right): \alpha=1,2, n=1, \ldots, r_{m}\left(\phi_{m}(O)\right)\right\},
$$

we can also find $\alpha \in\{1,2\}$ and $n \in\left\{1, \ldots, r_{m}\left(\phi_{m}(O)\right)\right\}$ so that

$$
y \in V_{m, n}^{\alpha}\left(\phi_{m}(O)\right) \text {. }
$$

Therefore, we see that

$$
(x, y) \in O \times V_{m, n}^{\alpha}\left(\phi_{m}(O)\right) \subset C_{m, n}^{\alpha},
$$

so that $\bigcup\left\{\mathscr{C}_{m, n}: m, n \in \mathbb{N}\right\}$ forms an open cover of $X \times Y$. By Lemma 1, we conclude that $X \times Y$ is weakly infinite-dimensional.

We single out two special cases of separate interest. In the second corollary, which we were unable to find in the literature, $I$ denotes the closed unit interval. 
Corollary 1. The product of $R$. Pol's uncountable-dimensional compact $C$-space with any weakly infinite-dimensional compactum is again weakly infinite-dimensional.

Corollary 2. $X$ is a weakly infinite-dimensional compactum if and only if $X \times I$ is a weakly infinite-dimensional compactum.

Question 1. If $X \times I$ is weakly infinite-dimensional, then must $X$ have property C?

In the final part of this note, we consider the following question.

Question 2. What properties must an infinite-dimensional space possess to ensure that its product with every zero-dimensional, hence countable-dimensional, space is a $C$-space (is weakly infinite-dimensional)?

Necessarily, such a space must itself be a $C$-space (weakly infinite-dimensional), but it is also known that this is not a sufficient condition for productivity with zero-dimensional factors [P]. On the other hand, as the following example shows, while we have shown that compactness of a $C$-space (weakly infinitedimensional) factor is a sufficient condition for such productivity, it is not a necessary condition.

Recall that R. Pol's compactum, when constructed as a subspace of the Hilbert cube, has the form $P=X \cup B_{1} \cup B_{2}$, where $X$ is a topologically complete, totally disconnected, strongly infinite-dimensional subspace of the Hilbert cube with countable-dimensional remainder $B_{1} \cup B_{2}=P / X$. So constructed, $B_{1}$ and $B_{2}$ are disjoint Bernstein sets; i.e., all compact subsets of $B_{1}$ and $B_{2}$ are countable [P2]. It is known that $B_{1} \cup X$ and $B_{2} \cup X$ are noncompact $C$-spaces [EP].

We will also need the following classical result.

Lemma 2 [E, 4.3.6]. If $f: X \rightarrow Y$ is a closed mapping between spaces $X$ and $Y$ where $\operatorname{dim} f^{-1}(y) \leq 0$ for each $y \in Y$, then $\operatorname{dim} X \leq \operatorname{dim} Y$.

Theorem 2. Given $B_{i}$, where $i \in\{1,2\}$, and $X$ as above, the product of $B_{i} \cup X$ with any zero-dimensional space $Z$ is a $C$-space and thus is weakly infinitedimensional.

Proof. The proof follows ideas of [EP] and [P2]. We assume, without loss of generality, that $i=1$, set $X_{1}=\left(B_{1} \cup X\right)$, and let $Z$ be any zero-dimensional space. We will show that $X_{1} \times Z$ has property $C$ as a subspace of $P \times Z$.

Given a sequence $\left\{\mathscr{U}_{n}: n \in \mathbb{N}\right\}$ of covers of $X_{1} \times Z$ by open subsets of $P \times Z$, for each fixed $n \in \mathbb{N}$ we set

$$
K_{n}=(P \times Z) \backslash \bigcup\left\{U: U \in \mathscr{U}_{n}\right\} .
$$

Each $K_{n}$ is a closed subset of $P \times Z$ contained entirely in $B_{2} \times Z$. Since the restricted projection $\pi: K_{n} \rightarrow Z$ is a closed mapping with countable, hence at most zero-dimensional, fibers, we apply Lemma 2 to see that $\operatorname{dim} K_{n} \leq 0$. 
Being a countable union of closed zero-dimensional sets,

$$
\operatorname{dim} \bigcup\left\{K_{n}: n \in \mathbb{N}\right\} \leq 0 \text {. }
$$

Thus, we can choose an at most zero-dimensional $G_{\delta}$-subset $A \subset P \times Z[\mathrm{E}$, 4.1.19] so that

$$
\bigcup\left\{K_{n}: n \in \mathbb{N}\right\} \subset A .
$$

Then, we also have

$$
\begin{aligned}
(P \times Z) \backslash A & \subset(P \times Z) \backslash \bigcup\left\{K_{n}: n \in \mathbb{N}\right\} \\
& \subset \bigcap\left\{(P \times Z) \backslash K_{n}: n \in \mathbb{N}\right\} \\
& \subset \bigcap\left\{\cup\left\{U: U \in \mathscr{U}_{n}\right\}: n \in \mathbb{N}\right\},
\end{aligned}
$$

and, in particular, for each $n \in \mathbb{N}$ we see that $\mathscr{U}_{n}$ is an open cover of $(P \times Z) \backslash A$ in $P \times Z$. Since $P$ is a compact $C$-space, $P \times Z$ is a $C$-space, and, being an $F_{\sigma}$ subset of $P \times Z,(P \times Z) \backslash A$ is also a $C$-space [AG]. Thus, we can choose a $C$-refinement $\mathscr{V}_{n}$ of $\mathscr{U}_{n}$ for each $n>1$ so that the $\bigcup\left\{\mathscr{V}_{n}: n>1\right\}$ is a cover of $(P \times Z) \backslash A$, hence also a cover of $\left(X_{1} \times Z\right) \backslash A$.

Finally, since $\left(X_{1} \times Z\right) \cap A \subset A$, we see that $\left(X_{1} \times Z\right) \cap A$ can be at most zerodimensional. Therefore, we can choose a $C$-refinement $\mathscr{V}_{1}$ of the remaining cover $\mathscr{U}_{1}$ so that $\mathscr{V}_{1}$ still covers $\left(X_{1} \times Z\right) \cap A$. Then, the $\bigcup\left\{\mathscr{V}_{n}: n \in \mathbb{N}\right\}$ is now a cover of all of $X_{1} \times Z$, which completes the proof that $X_{1} \times Z$ has property $C$.

\section{REFERENCES}

[AG] D. F. Addis and J. H. Gresham, A class of infinite-dimensional spaces. Part I: Dimension theory and Alexandroff's problem, Fund. Math. 101 (1978), 195-205.

[D] J. Dieudonné, Un critère de normalité pour les espaces prodults, Colloq. Math. 6 (1958), 29-32.

[E] R. Engelking, Dimension theory, North-Holland, Amsterdam, 1978.

[EP] R. Engelking and E. Pol, Countable-dimensional spaces: A survey, Dissertationes Math. 216 (1983), 1-41.

[P] E. Pol, $A$ weakly infinite-dimensional space whose product with the irrationals is strongly infinite-dimensional, Proc. Amer. Math. Soc. 98 (1986), 349-352.

[P1] R. Pol, $A$ weakly infinite-dimensional compactum which is not countable-dimensional, Proc. Amer. Math. Soc. 82 (1981), 634-636.

[P2] - A remark on A-weakly infinite-dimensional spaces, Topology Appl. 13 (1982), 97-101.

[R1] D. M. Rohm, Alternative characterizations of weak infinite-dimensionality and their relation to a problem of Alexandroff's, Ph.D. dissertation, Oregon State Univ., 1987.

[R2] _ Products of infinite-dimensional spaces, Proc. Amer. Math. Soc. (to appear).

Department of Mathematics and Computing, The University of Wisconsin-Stevens Point, Stevens Point, Wisconsin 54481 